BULLETIN (New Series) OF THE

AMERICAN MATHEMATICAL SOCIETY

Volume 43, Number 2, Pages 227-230

S 0273-0979(06)01114-1

Article electronically published on March 8, 2006

\title{
KNOWN AND UNKNOWN RESULTS ON ELLIPTIC BOUNDARY PROBLEMS
}

\author{
GERD GRUBB
}

In a survey article by W. N. Everitt and L. Markus [EM05] in the October 2005 issue of Bulletin AMS, the authors raise some questions on boundary problems for elliptic partial differential operators. The article is primarily concerned with ODE where the choice of boundary conditions is finite dimensional, but a secondary purpose is to extend their points of view to elliptic PDE where the possible boundary conditions range in infinite-dimensional spaces, and here the authors present a number of what they consider open problems.

The purpose of this note is to show how answers to the problems, as well as much more extensive results, can be found in the existing literature.

Section 2.2 of [EM05] presents the partial differential operator to be considered, namely the Laplacian $\Delta=\partial^{2} / \partial x_{1}^{2}+\partial^{2} / \partial x_{2}^{2}$ on the unit disk $\Omega=\left\{\left(x_{1}, x_{2}\right) \in \mathbb{R}^{2} \mid\right.$ $\left.x_{1}^{2}+x_{2}^{2}<1\right\}$. With $A$ denoting $-\Delta$ applied in the distribution sense, the authors consider several operators acting like $A$ (realizations of $A$ ):

- $T_{0}$ is the minimal operator, with domain $D\left(T_{0}\right)=\stackrel{\circ}{W}(\Omega)$,

- $T_{1}$ is the maximal operator, with domain $D\left(T_{1}\right)=\left\{u \in L_{2}(\Omega) \mid A u \in L_{2}(\Omega)\right\}$,

- $T_{\text {Dir }}$ is the Dirichlet realization, with domain $D\left(T_{\text {Dir }}\right)=W^{2}(\Omega) \cap \stackrel{\circ}{W^{1}}(\Omega)$.

It has been known for many years that $T_{\text {Dir }}$ is selfadjoint in $L_{2}(\Omega)$ with a positive lower bound, that $T_{0}$ is closed, densely defined and symmetric with the same lower bound, and that $T_{1}=T_{0}^{*}$. $T_{1}$ has an infinite-dimensional nullspace consisting of the harmonic functions in $L_{2}(\Omega)$,

$$
L_{2}^{\Delta}(\Omega)=\left\{u \in L_{2}(\Omega) \mid A u=0\right\} .
$$

In Section 2.3, the authors introduce one more realization, $T_{\mathrm{Har}}$, that they find mysterious; its domain is

$$
D\left(T_{\mathrm{Har}}\right)=\stackrel{\circ}{W^{2}}(\Omega) \dot{+} L_{2}^{\Delta}(\Omega),
$$

and it is selfadjoint. They prove by examples, referring to EMP05 for details, that $D\left(T_{\mathrm{Har}}\right)$ is not contained in $W^{1}(\Omega)$ and that there are elements of $D\left(T_{\mathrm{Har}}\right)$ that do not have pointwise radial limits for $r \nearrow 1$. They claim on p. 480:

"It is an unsolved problem as to whether the operator $T_{\mathrm{Har}}$, as a self-adjoint extension of the Laplace operator in the unit disk, is unique in some noteworthy

Received by the editors December 10, 2005, and, in revised form, January 19, 2006.

2000 Mathematics Subject Classification. Primary 35J25, 47B25; Secondary 35J67.

Key words and phrases. Elliptic boundary value problems, selfadjoint realizations, harmonic functions, Krein's soft extension.

(C)2006 American Mathematical Society Reverts to public domain 28 years from publication 
way. For instance, can $T_{\text {Har }}$ be characterized, among all self-adjoint operators for the Laplace differential expression, or even among some interesting subclass, by mathematically significant intrinsic properties?"

Answer 1. In the setting of abstract operators in Hilbert space, $T_{\mathrm{Har}}$ is the solution by J. von Neumann [N29] of the problem of finding a selfadjoint extension of a given symmetric, densely defined positive operator. It was found before the Friedrichs extension [F34, which in the present situation equals $T_{\text {Dir }}$. M. G. Krein showed in K47 that the full set of selfajoint nonnegative extensions of $T_{0}$ can be characterized as the operators lying between the "hard" extension (here $T_{\text {Dir }}$ ) and the "soft" extension (here $T_{\mathrm{Har}}$ ) in a sense defined via sesquilinear forms. So indeed $T_{\mathrm{Har}}$ is intrinsically characterized as Krein's soft extension of $T_{0}$.

In terms of boundary conditions, Grubb [G68] showed that $T_{\text {Har }}$ represents the nonlocal boundary condition

$$
M u=0, \text { where } M=\gamma_{1}-P \gamma_{0} ;
$$

here $\gamma_{j} u=\left.\partial_{n}^{j} u\right|_{\partial \Omega}$, the $j$ 'th normal derivative, and $P$ is the "Dirichlet-to-Neumann" operator over $\partial \Omega$, mapping $\gamma_{0} u$ into $\gamma_{1} u$ when $u \in L_{2}^{\Delta}(\Omega)$. These statements build on the result of Lions and Magenes from 1961-62, reproduced in the book [LM68, that the trace operators $\gamma_{j}$ extend to continuous mappings from $D\left(T_{1}\right)$ to $W^{-j-\frac{1}{2}}(\partial \Omega), j=0,1$. (Another source is to apply Hörmander H63, Th. 4.3.1, 2.5.6, to $L_{2}^{\Delta}(\Omega)$, "partial hypoellipticity at the boundary".) Vainberg and Grushin showed in VG67 that $P$ is a pseudodifferential operator of order 1 . This also follows from Seeley S66 and Hörmander [H66, and is accounted for in detail in Grubb G71. It is shown in G68 that $D\left(T_{\mathrm{Har}}\right) \nsubseteq W^{s}(\Omega)$ for all $s>0$.

Next, Everitt and Markus write on p. 480:

"The question of uniqueness for the operator $T_{\text {Har }}$ also leads to another problem. If $T_{\mathrm{Har}}$ is unique, can all other self-adjoint extensions of the Laplace operator on the unit disk be determined by applying generalized boundary conditions to functions in the maximal domain $D\left(T_{1}\right)$, on the boundary $\partial \Omega$, using the properties of the Trace Theorem?" (The Trace Theorem in EM03] defines $\gamma_{j}$ on $W^{l}(\Omega)$ for $l>j$.)

Answer 2. The full set of selfadjoint extensions of $T_{0}$ was characterized in G68 in terms of boundary conditions, using the extended trace theorem of LM68 indicated above. The selfadjoint extensions $\widetilde{T}$ correspond $1-1$ to the pairs $\{X, L\}$, where $X$ is a closed subspace of $W^{-\frac{1}{2}}(\partial \Omega)$ and $L$ is a selfadjoint operator from $X$ to the dual space $X^{\prime}$ (with $D(L) \subset X$ ). Here, when $\widetilde{T}$ corresponds to $\{X, L\}$, it represents the boundary condition

$$
\gamma_{0} u \in D(L),\left.\quad(M u)\right|_{X}=L \gamma_{0} u .
$$

The characterization is worked out for general elliptic operators on general smooth sets $\Omega$ and deals with many properties other than selfadjointness. The scale of semibounded realizations arising from sequilinear forms, as initiated by Krein, is considered in G70].

In Section 5 of EM05, the authors present a number of open problems. Most of these are concerned with ODE. The problem posed in PDE, Problem 5.7, is whether $T_{\mathrm{Har}}$ is globally determined when $\partial \Omega$ is connected. 
Comment 1. Realizations determined by purely differential boundary conditions are considered to be locally determined. As recalled above in Answer 1, $T_{\mathrm{Har}}$ is determined by the boundary condition (3). The operator $P$ is truly pseudodifferential; for the Laplace operator, its principal symbol is $-\left|\xi^{\prime}\right|$, and there are similar nonpolynomial symbols in more general cases (detailed formulas can be found in G71], Appendix). Then $P$ is in general nonlocal, with a distribution kernel not supported in the diagonal. In this sense, the boundary condition for $T_{\mathrm{Har}}$ is global.

Quotations from the end of the article, p. 498:

"... the Harmonic operator remains elusively unspecified by any kind of boundary evaluations."

Comment 2. No, it is determined by (3).

"Recent research (see [16]) has uncovered a continuum of distinct analogues of the operator $T_{\mathrm{Har}},[\ldots]$ each of which has a domain not entirely contained within the Sobolev space $W^{2}(\Omega)$. Hence these domains must contain some functions having no boundary values in the sense of the trace map [...].

"It remains an open problem to produce a coherent, and plausibly explicit, description of the set of all self-adjoint operators generated by the Laplace differential expression on the unit disk [...]."

Comment 3. No, the problem is not open; see Answer 2 above. All the functions in the maximal domain have boundary values in the sense of the extended trace map of LM68. Domains not contained in $W^{2}(\Omega)$ are abundant, in view of the $1-1$ correspondence between selfadjoint realizations $\widetilde{T}$ and arbitrary pairs $\{X, L\}$.

For a fuller picture, we include some further references that are relevant in this context. The grounds for the abstract part of the characterization were laid in Vishik V52 and Birman B56, in addition to K47]. Some of our results were rediscovered by Alonso and Simon [AS80. (addendum AS81]) and taken up again in connection with a spectral asymptotics question in G83.

\section{About the Author}

Gerd Grubb is a professor at the University of Copenhagen, Denmark. She has received the Hermer's Prize and the Marie Lønggaard Award, and is a docteur honoris causa at Université de Reims.

\section{REFERENCES}

[AS80] A. Alonso and B. Simon, The Birman-Krein-Vishik theory of self-adjoint extensions of semibounded operators, J. Operator Theory 4 (1980), 251-279. MR0595414 (81m:47038)

[AS81] A. Alonso and B. Simon, Addenda to "The Birman-Krein-Vishik theory of self-adjoint extensions of semibounded operators", J. Operator Theory 6 (1981), 407. MR0643699 (83a:47032)

[B56] M. S. Birman, On the theory of self-adjoint extensions of positive definite operators, Mat. Sb. 38: 80 (1956), 431-450 (Russian). MR0080271 (18:220d)

[EM03] W. N. Everitt and L. Markus, Elliptic partial differential operators and symplectic algebra, Memoirs of Amer. Math. Soc. 162 (2003), Number 770. MR.1955204 (2004d:47054)

[EM05] W. N. Everitt and L. Markus, Complex symplectic spaces and boundary value problems, Bull. Amer. Math. Soc. 42 (2005), 461-500. MR2163706 
[EMP05] W. N. Everitt, L. Markus and M. Plum, An unusual self-adjoint linear partial differential operator, Trans. Amer. Math. Soc. 357 (2005), 1303-1324. MR 2115367 (2005j:35038)

[F34] K. Friedrichs, Spektraltheorie halbbeschränkter Operatoren und Anwendung auf die Spektralzerlegung von Differentialoperatoren, Math. Ann. 109 (1934), 465-487. MR.1512905

[G68] G. Grubb, A characterization of the non-local boundary value problems associated with an elliptic operator, Ann. Scuola Norm. Sup. Pisa 22 (1968), 425-513, from Stanford University dissertation, 1966. MR0239269 (39:626)

[G70] G. Grubb, Les problèmes aux limites généraux d'un opérateur elliptique, provenant de la théorie variationnelle, Bull. Sci. Math. 94 (1970), 113-157, from Stanford University dissertation, 1966. MR0280866 (43:6585)

[G71] G. Grubb, On coerciveness and semiboundedness of general boundary problems, Isr. J. Math. 10 (1971), 32-95. MR0318665 (47:7212)

[G73] G. Grubb, Weakly semibounded boundary problems and sesquilinear forms, Ann. Inst. Fourier Grenoble 23 (1973), 145-194. MR0344669 (49:9408)

[G73a] G. Grubb, Semibounded boundary problems for elliptic operators, AMS Proc. Symp. Pure Math. 23 (1973), 113-123. MR0344668(49:9407)

[G74] G. Grubb, Properties of normal boundary problems for elliptic even-order systems, Ann. Scuola Norm. Sup. Pisa 1 (ser. IV) (1974), 1-61. MR0492833 (58:11895)

[G83] G. Grubb, Spectral asymptotics for the "soft" selfadjoint extension of a symmetric elliptic differential operator, J. Operator Theory 10 (1983), 9-20. MR.0715550 (84k:35107)

[H63] L. Hörmander, Linear Partial Differential Operators, Springer Verlag, Berlin, Heidelberg, 1963. MR0161012 (28:4221)

[H66] L. Hörmander, Pseudo-differential operators and non-elliptic boundary problems, Ann. Math. 83 (1966), 129-209. MR0233064 (38:1387)

[K47] M. G. Krein, Theory of self-adjoint extensions of symmetric semi-bounded operators and applications I, Mat. Sb. 20: 62 (1947), 431-495 (Russian). MR0024574 (9:515c)

[LM68] J.-L. Lions and E. Magenes, Problèmes aux limites non homogènes et applications, Vol. 1, Editions Dunod, Paris, 1968; English translation in Springer Grundlehren series, 1973. MR0247243(40:512)

[N29] J. von Neumann, Allgemeine Eigenwerttheorie Hermitescher Funktionaloperatoren, Math. Ann. 102 (1929), 49-131.

[S66] R. T. Seeley, Singular integrals and boundary value problems, Amer. J. Math. 88 (1966), 781-809. MR0209915 (35:810)

[VG67] B. R. Vainberg and V. V. Grusin, Uniformly non-elliptic problems II, Mat. Sb. 73 (1967), 126-154; Math. USSR-Sb. 2 (1967), 111-133. MR0217463(36:552)

[V52] M. I. Vishik, On general boundary value problems for elliptic differential equations, Trudy Moskov. Mat. Obsv. 1 (1952), 187-246; Amer. Math. Soc. Transl. 24 (1963), $107-172$.

Editor's note: Everitt and Markus have thanked Grubb for her help and advice following the publication of their survey paper in the October 2005 issue of the Bulletin. They regret that they inadvertently failed to give due recognition to Grubb's results. However, they point out that they have not been alone in this omission.

Department of Mathematics, University of Copenhagen, Universitetsparken 5, DK-2100 Copenhagen, Denmark

E-mail address: grubb@math.ku.dk 